\title{
All fibre Q-switched Thulium-doped fibre laser incorporating Thulium-Holmium co-doped fibre as a saturable absorber
}

Shi Li

Harbin Engineering University

Elfed Lewis

University of Limerick

Gerald Farrell

Technological University Dublin, gerald.farrell@tudublin.ie

See next page for additional authors

Follow this and additional works at: https://arrow.tudublin.ie/prcart

Part of the Electrical and Computer Engineering Commons, Engineering Physics Commons, and the Optics Commons

\section{Recommended Citation}

Shi Li, Yu Yin, Elfed Lewis, Gerald Garrell, Ahmad Haziq Aiman Rosol, A.A. Latiff, Sulaiman Wadi Harun, Bingang Guo, Pengfei Wang, All fibre Q-switched Thulium-doped fibre laser incorporating Thulium-Holmium co-doped fibre as a saturable absorber, Optics Communications, Volume 450, 2019, Pages 160-165, ISSN 0030-4018, DOI: 10.1016/j.optcom.2019.05.058.

This Article is brought to you for free and open access by the Photonics Research Centre at ARROW@TU Dublin. It has been accepted for inclusion in Articles by an authorized administrator of ARROW@TU Dublin. For more information, please contact arrow.admin@tudublin.ie, aisling.coyne@tudublin.ie,gerard.connolly@tudublin.ie. Funder: National Key R\&D Program of China; National Natural Science Foundation of China (NSFC); Harbin Engineering University

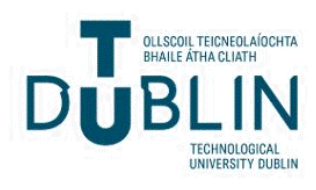




\section{Authors}

Shi Li, Elfed Lewis, Gerald Farrell, Ahmad Haziq Aiman Rosol, A.A. Latiff, Sulaiman Wadi Harun, Bingang Guo, and Pengfei Wang 


\title{
All fibre Q-switched Thulium-doped fibre laser incorporating Thulium-Holmium co-doped fibre as a saturable absorber
}

\author{
Shi Li ${ }^{\text {a }}$, Yu Yin ${ }^{\text {a }}$, Elfed Lewis ${ }^{\mathrm{b}}$, Gerald Garrell ${ }^{\mathrm{c}}$, Ahmad Haziq Aiman Rosol ${ }^{\mathrm{d}}$, A.A. Latiff ${ }^{\mathrm{d}}$, \\ Sulaiman Wadi Harun ${ }^{\mathrm{d}}$, Bingang Guo ${ }^{\mathrm{e}}$, Pengfei Wang ${ }^{\mathrm{a}, \mathrm{f}, *}$ \\ ${ }^{a}$ Key Laboratory of In-fibre Integrated Optics of Ministry of Education, College of Science, Harbin Engineering University, Harbin 150001, China \\ b Optical Fibre Sensors Research Centre, Department of Electronic and Computer Engineering, University of Limerick, Limerick, Ireland \\ c Photonics Research Centre, Technological University Dublin, Kevin Street, Dublin 8, Ireland \\ ${ }^{\mathrm{d}}$ Department of Electrical Engineering, University of Malaya, 50603, Kuala Lumpur, Malaysia \\ e Shenzhen Holokook Technology Co., Ltd, Shenzhen, 518000, China \\ ${ }^{\mathrm{f}}$ Key Laboratory of Optoelectronic Devices and Systems of Ministry of Education and Guangdong Province, College of Optoelectronic Engineering, Shenzhen \\ University, Shenzhen, 518060, China
}

\section{A R T I C L E I N F O}

\section{MSC:}

$00-01$

99-00

Keywords:

Fibre laser

Q-switched pulse

Doped fibre saturable absorber

\begin{abstract}
A B S T R A C T
A novel all fibre Q-switched Thulium-doped fibre laser (TDFL) is reported which includes a short length of a Thulium-Holmium co-doped fibre (THDF) as a saturable absorber. A high repetition rate $(27.26 \mathrm{kHz}) \mathrm{coupled}$ with a low pulse width $(19.06 \mu \mathrm{s})$ is obtained for single wavelength Q-switched pulse operation at an output wavelength of $1911.5 \mathrm{~nm}$ using a pump power of $200 \mathrm{~mW}$. Increasing the pump power from $200 \mathrm{~mW}$ to 700 $\mathrm{mW}$ results in the repetition rate increasing from $27.26 \mathrm{kHz}$ to $99.67 \mathrm{kHz}$ and the pulse width decreasing from $19.06 \mu$ s to $920 \mathrm{~ns}$. The centre wavelength of the single Q-switched pulse was also red shifted from $1911.5 \mathrm{~nm}$ to $1932.5 \mathrm{~nm}$ with increasing the pump power. A $45 \mathrm{~m}$ length single-mode fibre (SMF-28) provided dispersion compensation, and effectively an SMF-THDF-SMF structure is inserted in the cavity which operates in a similar manner to an SMF-MMF-SMF structure, providing a strong multimode interference effect which supports dualwavelength operation. A stable dual-wavelength Q-switched pulse was achieved at a threshold pump power of $213 \mathrm{~mW}$. The dual-wavelength Q-switched pulse operation was generated at $1911.5 \mathrm{~nm}$ and $1914.5 \mathrm{~nm}$ with a repetition rate of $8.45 \mathrm{kHz}$ and pulse width of $20.02 \mu \mathrm{s}$. The dual-wavelength spacing of this pulse operation was $3 \mathrm{~nm}$, which was in good agreement with calculations based on the multimode interference effect induced by the THDF. The repetition rate increased from $8.45 \mathrm{kHz}$ to $70.65 \mathrm{kHz}$ and the pulse width decreased from $20.02 \mu \mathrm{s}$ to $870 \mathrm{~ns}$ with increasing pump power. At the maximum pump power of $700 \mathrm{~mW}$, the maximum output power was measured as $27.4 \mathrm{~mW}$. The experimental results confirm that the THDF can be utilized as a SA to generate a stable and tunable single-wavelength Q-switched pulse output as well as dual-wavelength Q-switched pulse in the $2.0 \mu \mathrm{m}$ wavelength region.
\end{abstract}

\section{Introduction}

During the past few decades, pulse fibre lasers have developed rapidly in numerous application fields including sensing, spectral ranging, optical communications, medical treatment and material processing etc. [1]. Q-switched fibre lasers have attracted significant attention particularly in applications where only a low repetition rate is required [2]. Furthermore, Q-switched fibre laser research has also expanded to cover multiple wavelength bands including those centred on $1.0 \mu \mathrm{m}, 1.5 \mu \mathrm{m}$ and $2.0 \mu \mathrm{m}$ [3-5]. Among these lasing bands, fibre lasers in the $2.0 \mu \mathrm{m}$ wavelength range have been successfully developed and are attractive for use in many applications including fibre sensing and optical communications due to their inherent advantages of being eye safe and being suitable for low loss atmospheric transmission applications [6-8].

Numerous approaches have been reported for the generation of Q-switched pulsed laser sources including the nonlinear optical loop mirror (NOLM) [9], nonlinear polarization rotation (NPR) [10] and through the use of saturable absorbers (SAs). The passive Q-switched technique based on SAs is considered one of the most efficient methods for pulse generation. Semiconductor saturable absorber mirrors (SESAMs) have proved to be a popular means of achieving passively Q-switched fibre lasers. However, they exhibit a low damage threshold, narrow bandwidth and high cost which have collectively hindered their

\footnotetext{
* Corresponding author.

E-mail address: pengfei.wang@dit.ie (P. Wang).
} 
development. To date, a series of nanomaterials have also been developed for Q-switched pulse generation including carbon nanotubes [11, 12], graphene [13,14], topological insulators [15], metal nanoparticles $[3,16]$, transition metal dichalcogenides $[4,17,18]$ and black phosphorus [19]. However, these nanomaterials still exhibit numerous drawbacks, including high cost, difficult fabrication, non-uniformity etc. Therefore, recent research efforts have largely concentrated around the use of SAs for Q-switched pulse generation. Gain medium fibres have also attracted strong interest due to their stable absorption profile. These doped fibres can play a similar role within the fibre laser cavity to SAs due to energy level transition of the rare earth ions. Given the wide range of possible doped fibre types, there exists a large number of different available processing techniques which in turn results in different absorption coefficient ranges. However, their absorption characteristics can be optimized by suitable predesign, where the simple and common method simply comprises adjustment of the doped fibre length.

Doped fibre based SA has excellent stability and this, coupled with optimized absorption means that they have been widely investigated in recent literature. In 2010, Tsai et al. demonstrated a $2 \mathrm{kHz}$ repetition rate Q-switched EDFL incorporating a Thulium-doped fibre (TDF) for the first time [20]. In 2012, Tao et al. demonstrated a thuliumholmium-doped fibre (THDF) based EDFL which was Q-switched and mode-locked [21], which further confirmed the possibility of use of a doped fibre as an SA in fibre lasers. Recently, Latiff et al. have successfully demonstrated an all-fibre dual-wavelength Q-switched and mode-locked EDFL including a short length of THDF in the laser cavity [22]. However, reported investigations of doped-fibre in the $2.0 \mu \mathrm{m}$ laser range remains rare. There therefore exists a clear need for and strong motivation to investigate alternative wavelength regions based on the use of a doped-fibre saturable absorber.

In this paper, an $18 \mathrm{~cm}$ long THDF was fusion spliced with singlemode fibre (SMF-28) to form a SMF-THDF-SMF structure. The SMFTHDF-SMF structure works in a similar fashion to a multi-mode fibre (MMF) based SMF-MMF-SMF structure placed within the lasing cavity [22]. A stable tunable single wavelength Q-switched pulse laser output was obtained using the doped fibre structure in the lasing cavity. Furthermore, a $45 \mathrm{~m}$ long SMF (SMF-28) was inserted into the lasing cavity to optimize the in-cavity dispersion. A stable dual-wavelength Qswitched pulse was thus obtained, whose peak spacing agrees well with the theoretically predicted value owing to the multimode interference effect induced by the SMF-THDF-SMF structure. To the best of our knowledge, this is the first time that a tunable single Q-switched and dual-wavelength pulse operation laser has been experimentally demonstrated where a length of THDF is used as the SA in a Thulium-doped fibre laser (TDFL).

\section{Experimental installation and processing}

The THDF was fusion spliced with two standard SMFs to form the SMF-THDF-SMF structure. The single-clad THDF was $18 \mathrm{~cm}$ in length with core and cladding diameters of $11.5 \mu \mathrm{m}$ and $125 \mu \mathrm{m}$ respectively. The refractive index difference and cutoff wavelength were 0.0068 and $2545 \mathrm{~nm}$ respectively. The SMF-THDF-SMF structure is shown schematically in Fig. 1(a). The cutoff wavelength values of the THDF and SMF fibres are $2545 \mathrm{~nm}$ and $1260 \mathrm{~nm}$, respectively. It functions as a SA device due to the energy level transition of the ions in the THDF, which is similar to the Pauli blocking principle which occurs in a two-dimensional material SA [23]. The operation of the THDF SA provides the basis upon which pulse operation is achieved in the designed fibre laser cavity. The THDF plays the same role as the length of multimode fibre within an SMF-MMF-SMF structure as the THDF in the SMF-THDF-SMF structure in this investigation, in that multimode interference occurs within the THDF section. According to the multimode interference effect theory within a fibre heterostructure, a series

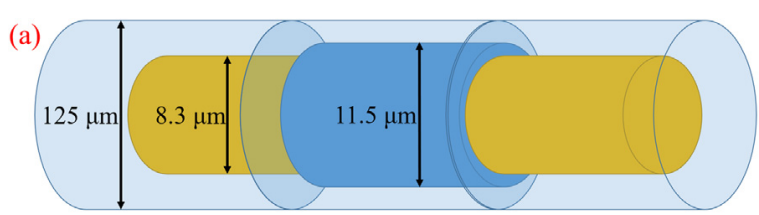

(b)

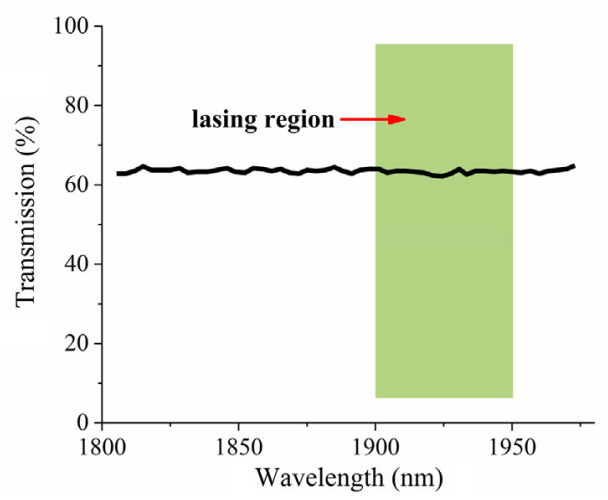

Fig. 1. SMF-THDF-SMF structure. (a) Schematic diagram of the structure with and $18 \mathrm{~cm}$ length of THDF (b) The spectral absorption characteristic of the THDF in the range 1800 to $1950 \mathrm{~nm}$.

of peak wavelengths are generated due to the multimode interference effect, which are given as [24]:

$\lambda_{0}=\frac{p n_{M M F} D_{M M F}^{2}}{L}, p=0,1,2,3 \ldots$,

where $n_{M M F}$ is the refractive index, $D_{M M F}$ is the diameter of the MMF core, and $L$ is the MMF length, respectively. $p$ is the self-imaging number. The SMF-THDF-SMF structure inherently generates a multimode interference effect, which in turn results in more than one peak wavelengths which provides the possibility of dual-wavelength output lasing operation [22]. The dual-wavelength lasing spacing can be calculated according to the following equation:

$\Delta \lambda=\frac{\lambda_{1} \lambda_{2}}{\Delta n L}$

where $\Delta \lambda$ is the dual-wavelength lasing spacing, $\lambda_{1}$ and $\lambda_{2}$ are the dualwavelength values, $\Delta n$ is the refractive index difference and $L$ is the length of the THDF.

In the SMF-THDF-SMF structure, the THDF length was chosen as $18 \mathrm{~cm}$ to ensure good absorption in the lasing region. The spectral absorption of the THDF was measured using a supercontinuum source (YSL SC-series) and an optical spectral analyzer (YOKOGAWA, AQ6370C). The absorption characteristic is shown in Fig. 1(b). Fig. 1(b) shows that the fibre has a transmission of $62.7 \%$ in the lasing region.

The balanced twin-detector method was used to measure the nonlinear transmission characteristic of the THDF [25]. The schematic diagram of the nonlinear transmission measurement is shown in Fig. 2(a). A home-made watt-level CW Tm-doped fibre laser and a acoustic optical modulator (AOM, $100 \mathrm{~ns}, 10 \mathrm{kHz}$ ) were used as the pump source. The nonlinear transmission of the film was measured by comparing the input power and output power values using a two-channel power meter (PM320E). As illustrated in Fig. 2(b), the THDF exhibits a classical saturable absorption characteristic in that the transmission initially increases with the pumping power and then levels out. The modulation depth of the THDF were measured as $36.2 \%$, respectively and the former is shown in Fig. 2(b). The nonsaturable loss is about $27.2 \%$ and the peak power intensity of the saturable point is about $60 \mathrm{~W}$. However, when the pumping power was set at low power level (below $60 \mathrm{~mW}$ ), a subtle drift of wavelength is generated when the pumping light propagate through the THDF [26]. The throughput instability is caused by the subtle drift of wavelength, which results in an unstable and hence 


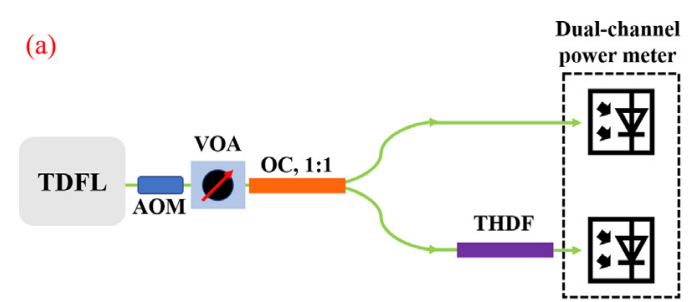

(b)

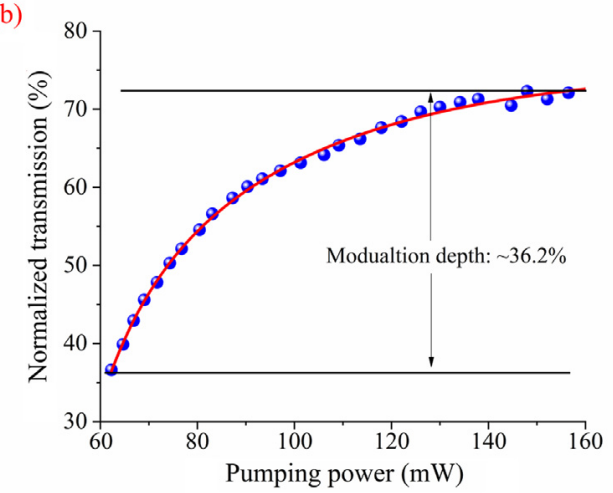

Fig. 2. (a) Schematic diagram of the nonlinear transmission measurement (balanced twin-detector method) (b) Nonlinear transmission of the THDF as a function of the pump power at wavelength of $1923 \mathrm{~nm}$.

unreliable measurement state when the pump power is low (below 60 $\mathrm{mW}$ ). Therefore, to avoid measurement error, the experimental results of the nonlinear transmission characteristic were measured only when the pump power was greater than $60 \mathrm{~mW}$. The experimental results are shown in Fig. 2(b).

Fig. 3 shows the schematic diagram of the Q-switched TDFL with THDF based SA. It consists of a tunable laser (Santec TSI-710) with a centre wavelength of $1562 \mathrm{~nm}$, an Erbium-doped fibre amplifier (EDFA, MC-EDFA-230), a wavelength division multiplexer (WDM, MCWDM15/19), a polarization independent isolator (PI-ISO, MCI-1980), a Thulium-doped fibre (Nufern SM-TSF-9/125) of $5 \mathrm{~m}$ length and a 1:9 optical coupler (OC, 1980-FBT-C). The pump source with a centre wavelength of $1562 \mathrm{~nm}$ was optically coupled into the EDFA which produced an amplified light output signal. The EDFA provides a maximum output power of $700 \mathrm{~mW}$, which was used to pump the gain medium, the $5 \mathrm{~m}$ long TDF via the WDM. The generated photons then propagate into the polarization independent isolator (PI-ISO) pigtailed with the THDF. The SMF has an $8.3 \mu \mathrm{m}$ core diameter. The THDF was used as the SA device, which generates a Q-switched pulse train. In the designed laser cavity, the PI-ISO not only kept the lasing unidirectional in operation, but also reduced Brillouin back-scattering, which otherwise could potentially disturb the stability of the pulsed operation. The $90 \%$ port of the optical coupler was spliced with the WDM to complete the ring cavity. The $10 \%$ port was connected to the optical spectral analyzer (OSA, YOKOGAWA, AQ-6370C) to measure the laser's output spectrum. The time resolved pulsed laser output signal was also measured using a digital storage Oscilloscope (Tektronix MDO4054-6, $6 \mathrm{GHz}$ ) and a photodetector (Kemai, PDA, $10 \mathrm{GHz}$ ). An optical power meter (Newport 1918-R) was used to measure the output power. The total cavity length of the fibre laser was about $23 \mathrm{~m}$.

\section{Results and discussion}

A wide range of experimental measurements were performed to fully characterize the optical source of this investigation. They are described in the following sub sections:

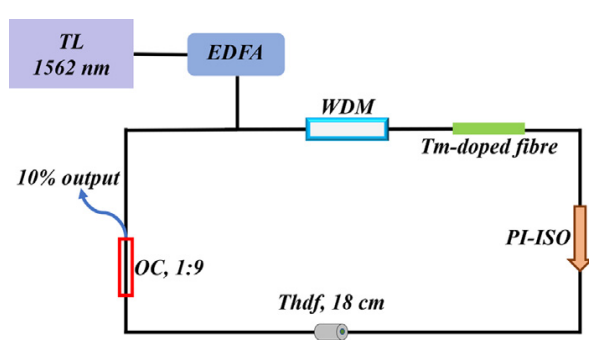

Fig. 3. Schematic diagram of the experimental setup.

3.1. Wavelength and time domain (pulse) characteristics of the single wavelength Q-switched device with a fixed (200 $\mathrm{mW}$ ) pump power

The characteristic of the single-wavelength Q-switched pulse operation is shown in Fig. 4. The Q-switched pulse was obtained by simply incorporating the $18 \mathrm{~cm}$ THDF into the standard cavity. The pump power was increased gradually from 0 and Continuous Wave (CW) lasing was observed when the pump power reached circa 100 $\mathrm{mW}$. There was no evidence of any pulse-like behaviour in the timebased waveforms observed on the oscilloscope. As the power was further increased to $200 \mathrm{~mW}$, Q-switching operation was initiated and recorded. The output spectrum and typical pulse train waveform are shown in Fig. 4(a) and (b) respectively. The centre wavelength of the Qswitched laser output was $1911.5 \mathrm{~nm}$. The repetition rate and the pulse width were $27.26 \mathrm{kHz}$ and $19.06 \mu$ s respectively. In the lasing cavity, the $\mathrm{Tm}^{3+}$ ion in the THDF was excited from the ground state ${ }^{3} \mathrm{H}_{6}$ to the excited state ${ }^{3} F_{4}$ when the photons have sufficient energy to reach the bonding energy of the $\mathrm{Tm}^{3+}$. Once sufficient energy is reached at the ${ }^{3} \mathrm{~F}_{4}$ energy level of $\mathrm{Tm}^{3+}$, energy was transferred to the ${ }^{5} \mathrm{I}_{7}$ energy state of $\mathrm{Ho}^{3+}[23,27]$. According to the previously reported work, the lifetime of the $\mathrm{Tm}^{3+}$ is about $200 \mu$ s at the energy state of ${ }^{3} F_{4}$ [23]. The energy transition between the $\mathrm{Tm}^{3+}$ and $\mathrm{Ho}^{3+}$ in the THDF is very important as it underpins the generation of pulses and increases the repetition rate of the pulse operation. The interaction of the two ions in the THDF is similar to the Pauli blocking principle, which occurs in a two-dimensional SA material. In order to confirm the long-term stability of the Q-switched pulse operation, the emission spectra of the $\mathrm{Q}$-switched lasing were recorded every $1 \mathrm{~h}$ for $4 \mathrm{~h}$. The spectra recorded over the $4 \mathrm{~h}$ duration are shown in Fig. 4(c), and it was observed that neither the central wavelength nor output power values varied. These results clearly indicate that the Q-switched lasing operation was very stable.

3.2. Wavelength and time domain (pulse) characteristics of the single wavelength $q$-switched device with a varying the pump power

The performance of the single wavelength Q-switched TDFL was further investigated where a series of spectra were recorded (shown in Fig. 5(a)) where the pump power was increased from $200 \mathrm{~mW}$ to the maximum value of $700 \mathrm{~mW}$. It is worth noting that the centre wavelength of the Q-switched lasing was significantly red shifted when the pump power was increased. The observed red shifted wavelength value was approximately $4 \mathrm{~nm}$ for every $100 \mathrm{~mW}$ increase in the pump power. The THDF is a multi-mode fibre in the laser cavity, therefore the lasing modes of $L P_{01}$ and $L P_{11}$ may be excited at the SM-MM splicing point. There may be two modes, $n_{e f 01}$ and $n_{e f 11}$, propagating in the THDF fibre. The light may be coupled back into the single-mode fibre following transmission through the second SM-MM splicing point. At this point, the electric field is the sum of these two fields, which can be expressed by the following equation:

$E=k_{01} \exp \left(i \varphi_{01}\right)+k_{11} \gamma \exp \left(i \varphi_{11}\right)$ 

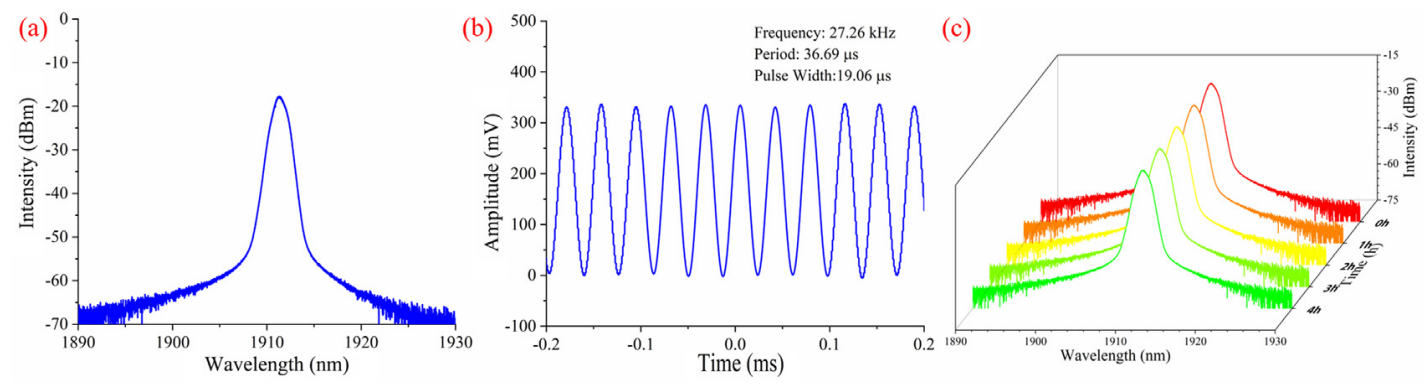

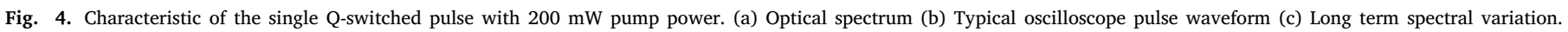

$k_{01}$ and $k_{11}$ are the fractions of the two electric fields coupled into the single-mode fibre, and is the excitation coefficient of the high-order mode. Accordingly, the intensity of the electric field is

$T(\lambda)=k_{01}^{2}+\gamma^{2} k_{11}^{2}+2 \gamma k_{01} k_{11} \cos \left[\frac{2 \pi L_{s}\left(n_{e f 01}-n_{e f 11}\right)}{\lambda}\right]$

$L_{s}$ is the length of the THDF and $\lambda$ is the laser wavelength.

In this laser cavity, the gain $G(\lambda)$ of the light signal is determined by two main sections: the emission of the active fibre, Tm-doped fibre and the absorption of the THDF, according to the equation below [26]:

$G(\lambda)=4.343\left[L_{a} N_{a} M_{a}\left[n_{a}\left(\sigma_{a}(\lambda)+\sigma_{e}(\lambda)\right)-\sigma_{a}(\lambda)\right]-L_{s} N_{s} M_{s} n_{s} \sigma_{a t}(\lambda)\right]$

where $L_{a}$ and $L_{s}$ are the length of the active fibre and THDF, $N_{a}$ and $N_{s}$ are the doping concentrations of the active fibre and the THDF. $M_{a}$ and $M_{s}$ are the overlap factors of the active fibre and THDF and $\sigma_{a}$ and $\sigma_{e}$ are the absorption and emission cross sections of the active fibre respectively. $\sigma_{a t}$ is the absorption cross section of the THDF at the wavelength of $\lambda . n_{a}$ is the fraction of the excited population at the ${ }^{3} F_{4}$ energy level of $T m$ ions, while $n_{s}$ is the fraction of the ground state population at the ${ }^{5} I_{7}$ energy level of $H o$ ions. $n_{a}$ is determined by the pump power, which shows a monotonic decreasing relationship with the pump power [27].

For the laser cavity described in this paper, the output lasing spectrum $O(\lambda)$ is determined by the combined interaction of the transmission spectrum of the filter, the emission spectrum of the gain fibre at different pump powers and the absorption spectrum of the THDF at different lasing intensities. Therefore, the $O(\lambda)$ can be written as follows:

$O(\lambda)=G(\lambda) T(\lambda)$

In (6), the centre wavelength location is determined by $G(\lambda)$, to be precise, mainly determined by $n_{a}$. $n_{a}$ shows a typical monotonic decreasing relationship with the pump power, it can explain that the centre lasing wavelength presents a significant shift to the long wavelength region.

The repetition rate, pulse width and output power were also recorded. It can be seen from Fig. 5(b) that when the pump power increased from the Q-switched threshold $200 \mathrm{~mW}$ to the maximum 700 $\mathrm{mW}$, the repetition rate increased from $27.26 \mathrm{kHz}$ to $99.67 \mathrm{kHz}$ and the pulse width decreased from $19.06 \mu$ s to $920 \mathrm{~ns}$, and thus exhibits the characteristic features of passive Q-switching $[28,29]$. These experimental results confirmed that the THDF used as a SA material in a fibre laser has the ability to generate a tunable Q-switched pulse operation. The output power was measured using the same optical power meter as referred to above and is shown in Fig. 5(c). The maximum output power is about $31.2 \mathrm{~mW}$.

\subsection{Wavelength and time domain (pulse) characteristics of the two wave- length q-switched device}

A $45 \mathrm{~m}$ length SMF was inserted into the laser cavity between the $18 \mathrm{~cm}$ length of THDF and the 1:9 optical coupler, increasing the total laser cavity length to $68 \mathrm{~m}$. The pump power was increased gradually, and CW lasing was obtained when the pumping power reached 105 $\mathrm{mW}$. The slightly higher threshold value was due to the added insertion loss caused by inclusion of the SMF. As the pump power was increased further to $213 \mathrm{~mW}$, a dual-wavelength Q-switched output was observed. The typical spectrum and oscilloscope pulse waveform recorded using the OSA and OSC are shown in Fig. 5(a) and (b), respectively. The two peaks of the dual-wavelength are located at $1911.5 \mathrm{~nm}$ and $1914.5 \mathrm{~nm}$. The repetition rate and pulse width were measured as $8.45 \mathrm{kHz}$ and $20.02 \mu$ sespectively. It is worth noting that the spacing of the two lasing peaks is about $3 \mathrm{~nm}$, which is in good agreement with the multimode interference effect of the THDF, based on (2). By increasing the pump power, it was observed that the repetition rate and pulse width of the dual-wavelength Q-switched pulse also changed. This agrees well with the characteristic features of passive Q-switching $[28,29]$. The repetition rate increased from $8.45 \mathrm{kHz}$ to $70.65 \mathrm{kHz}$ while the pulse width decreased from $20.02 \mu$ s to $870 \mathrm{~ns}$ when the pump power was increased from the Q-switched threshold of $213 \mathrm{~mW}$ to the maximum of $700 \mathrm{~mW}$. The output power was measured using the same optical power meter (Newport 1918-R), and the output power versus pump power is shown in Fig. 6(d). The maximum output power is about $27.4 \mathrm{~mW}$.

\section{Conclusion}

Typical tunable single-wavelength Q-switched operation was successfully obtained using the standard all-fibre laser cavity incorporating an $18 \mathrm{~cm}$ length of THDF as a SA, fabricated as part of this investigation. The energy level transition of the $\mathrm{Tm}^{3+}$ and $\mathrm{Ho}^{3+}$ in the THDF provides the saturable absorption to achieve Q-switched pulse operation. The typical tunable Q-switched laser action was generated with a single lasing peak at $1911.5 \mathrm{~nm}$ when a pump threshold power of $200 \mathrm{~mW}$ was reached. A red shift of $4 \mathrm{~nm}$ was observed with each subsequent $100 \mathrm{~mW}$ increase in the pump power. The repetition rate increased and the pulse width decreased with increasing pump power, which is in agreement with the typical characteristics of passive Q-switching. Dual-wavelength Q-switched pulse operation was also achieved by inserting a $45 \mathrm{~m}$ length of standard SMF into the same ring cavity. Dual-wavelength Q-switched lasing was obtained at the pump power of $213 \mathrm{~mW}$. The dual-wavelength Q-switched pulse operation was generated at $1911.5 \mathrm{~nm}$ and $1914.5 \mathrm{~nm}$ with a repetition rate of $8.45 \mathrm{kHz}$ and pulse width of $20.02 \mu \mathrm{s}$ at a pump power of $213 \mathrm{~mW}$. The SMF-THDF-SMF structure operates in a similar manner to an SMFMMF-SMF structure, which provides a strong multimode interference effect to support dual-wavelength operation. The experimental results obtained in this investigation confirm the potential of the THDF for use in many fibre laser applications. Furthermore, they also provide confirmation that the gain medium fibre can be usefully deployed in other application areas. 

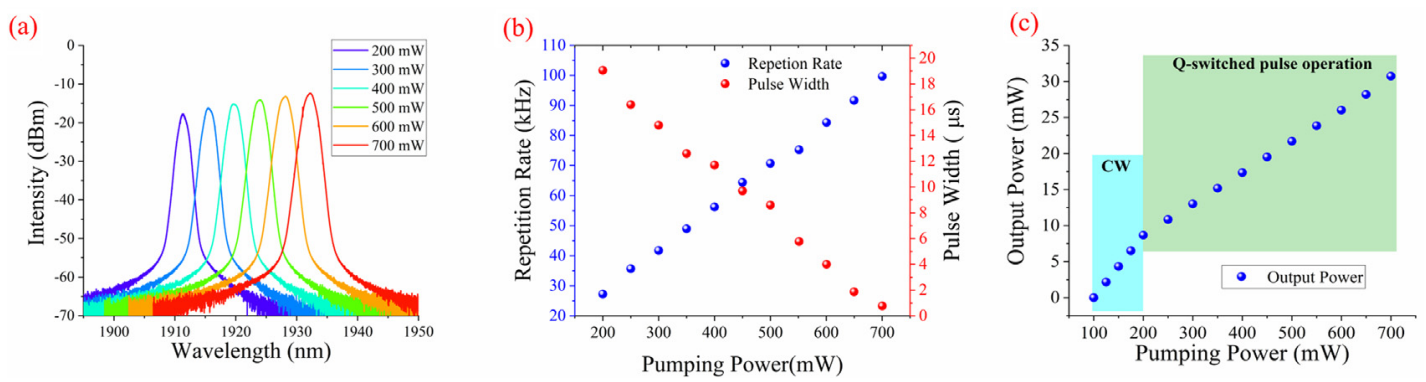

Fig. 5. Characteristic of the THDF Q-switched fibre laser versus the pump power. (a) Tunable spectra. (b) Repetition rate and pulse width. (c) Output power.

(a)

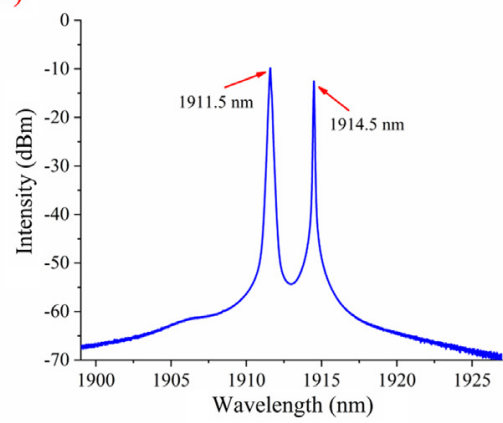

(c)

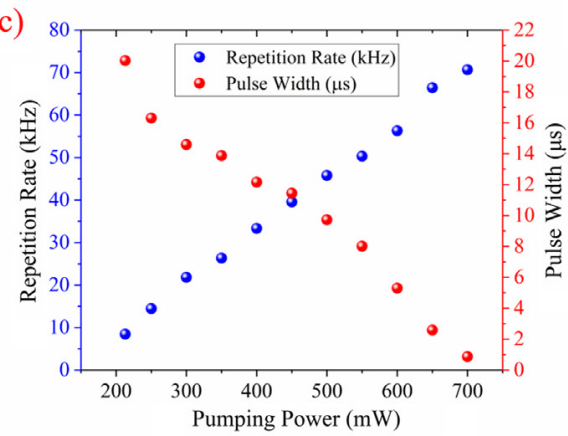

(b)

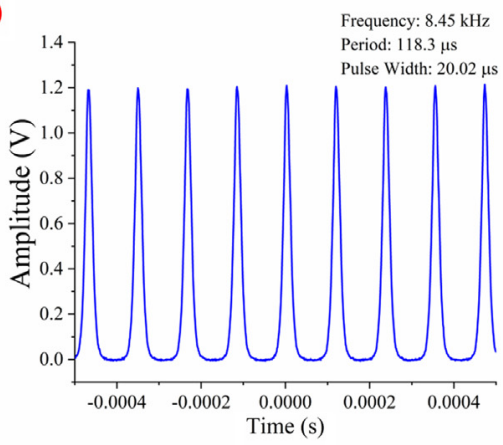

(d)

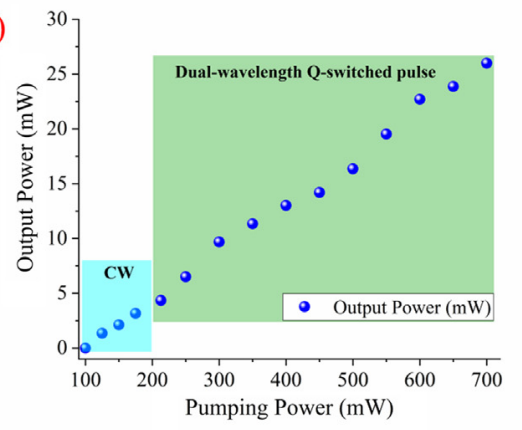

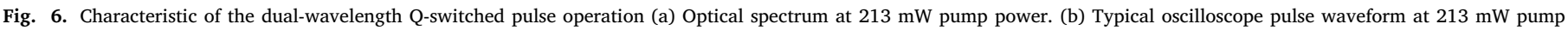
power. (c) Repetition rate and pulse width. Versus pump power (d) Output power versus pump power.

\section{Funding}

The work was supported by the National Key R\&D Program of China under grant 2016YFE0126500; National Natural Science Foundation of China (NSFC) under grant 61575050; The fundamental research funds for the central universities under grant HEUCFG201841; Key Program for Natural Science Foundation of Heilongjiang Province of China under grant ZD2016012; Open Fund of the State Key Laboratory on Integrated Optoelectronics under Grant IOSKL2016 KF03, this work was also supported by the 111 project (B13015) to the Harbin Engineering University.

\section{References}

[1] U. Keller, Recent developments in compact ultrafast lasers, Nature 424 (2003) 831, http://dx.doi.org/10.1038/nature01938.

[2] K. Bremer, A. Pal, S. Yao, E. Lewis, R. Sen, T. Sun, K. Grattan, Sensitive detection of CO 2 implementing tunable thulium-doped all-fiber laser, Appl. Opt. 52 (2013) 3957-3963, http://dx.doi.org/10.1364/ao.52.003957.

[3] H. Zhang, J. Liu, Gold nanobipyramids as saturable absorbers for passivelyQswitched laser generation in the $1.1 \mu \mathrm{m}$ region, Opt. Lett. 41 (2016) 1150-1152, http://dx.doi.org/10.1364/ol.41.001150.
[4] S.H. Kassani, R. Khazaeinezhad, H. Jeong, T. Nazari, D.-I. Yeom, K. Oh, All-fiber Er-doped Q-switched laser based on tungsten disulfide saturable absorber, Opt. Mater. Express 5 (2015) 373-379, http://dx.doi.org/10.1364/OME.5.000373.

[5] R. Woodward, R. Howe, T. Runcorn, G. Hu, F. Torrisi, E. Kelleher, T. Hasan, Wideband saturable absorption in few-layer molybdenum diselenide (MoSe 2) for Q-switching Yb-, Er-and Tm-doped fiber lasers, Opt. Express 23 (2015) 20051-20061, http://dx.doi.org/10.1364/oe.23.020051.

[6] Z. Li, A. Heidt, J. Daniel, Y. Jung, S. Alam, D.J. Richardson, Thulium-doped fiber amplifier for optical communications at $2 \mu \mathrm{m}$, Opt. Express 21 (2013) 9289-9297, http://dx.doi.org/10.1364/oe.21.009289.

[7] R. Soref, Group IV photonics: Enabling $2 \mu \mathrm{m}$ communications, Nat. Photon. 9 (2015) 358, http://dx.doi.org/10.1038/nphoton.2015.87.

[8] Y. Meng, Y. Li, Y. Xu, F. Wang, Carbon nanotube mode-locked thulium fiber laser with $200 \mathrm{~nm}$ tuning range, Sci. Rep. 7 (2017) 45109, http://dx.doi.org/ $10.1038 /$ srep45109.

[9] S. Chernikov, J. Taylor, Multigigabit/s pulse source based on the switching of an optical beat signal in a nonlinear fibre loop mirror, Electron. Lett. 29 (1993) 658-660, http://dx.doi.org/10.1049/el:19930441.

[10] X. Yang, C. Yang, Q-switched mode-locking in an erbium-doped femtosecond fiber laser based on nonlinear polarization rotation, Laser Phys. 19 (2009) 2106-2109, http://dx.doi.org/10.1134/s1054660x09210142.

[11] D.-P. Zhou, L. Wei, B. Dong, W.-K. Liu, Tunable passively $Q$-switched erbiumdoped fiber laser with carbon nanotubes as a saturable absorber, IEEE Photon. Technol. Lett. 22 (2010) 9-11, http://dx.doi.org/10.1109/LPT.2009.2035325.

[12] S. Harun, M. Ismail, F. Ahmad, M. Ismail, R. Nor, N. Zulkepely, H. Ahmad, A Q-switched erbium-doped fiber laser with a carbon nanotube based saturable absorber, Chin. Phys. Lett. 29 (2012) 114202, http://dx.doi.org/10.1088/16122011/10/2/025102. 
[13] Z. Luo, M. Zhou, J. Weng, G. Huang, H. Xu, C. Ye, Z. Cai, Graphene-based passively Q-switched dual-wavelength erbium-doped fiber laser, Opt. Lett. 35 (2010) 3709-3711, http://dx.doi.org/10.1364/OL.35.003709.

[14] J. Liu, S. Wu, Q.-H. Yang, P. Wang, Stable nanosecond pulse generation from a graphene-based passively Q-switched Yb-doped fiber laser, Opt. Lett. 36 (2011) 4008-4010, http://dx.doi.org/10.1364/OL.36.004008.

[15] Y. Chen, C. Zhao, S. Chen, J. Du, P. Tang, G. Jiang, H. Zhang, S. Wen, D. Tang, Large energy, wavelength widely tunable, topological insulator Qswitched erbium-doped fiber laser, IEEE J. Sel. Top. Quantum Electron. 20 (2014) 315-322, http://dx.doi.org/10.1109/jstqe.2013.2295196.

[16] D. Wu, J. Peng, Z. Cai, J. Weng, Z. Luo, N. Chen, H. Xu, Gold nanoparticles as a saturable absorber for visible $635 \mathrm{~nm}$ Q-switched pulse generation, Opt. Express 23 (2015) 24071-24076, http://dx.doi.org/10.1364/OE.23.024071.

[17] Z. Luo, Y. Huang, M. Zhong, Y. Li, J. Wu, B. Xu, H. Xu, Z. Cai, J. Peng, J. Weng, 1-, 1.5-, and 2-m fiber lasers Q-switched by a broadband few-layer MoS 2 saturable absorber, J. Lightwave Technol. 32 (2014) 4077-4084, http: //dx.doi.org/10.1109/JLT.2014.2362147.

[18] B. Chen, X. Zhang, K. Wu, H. Wang, J. Wang, J. Chen, Q-switched fiber laser based on transition metal dichalcogenides MoS 2, MoSe 2, WS 2, and WSe 2, Opt. Express 23 (2015) 26723-26737, http://dx.doi.org/10.1364/OE.23.026723.

[19] J. Li, H. Luo, B. Zhai, R. Lu, Z. Guo, H. Zhang, Y. Liu, Black phosphorus: a two-dimension saturable absorption material for mid-infrared Q-switched and mode-locked fiber lasers, Sci. Rep. 6 (2016) 30361, http://dx.doi.org/10.1038/ srep30361.

[20] S.H. Hung, T.Y. Tsai, Y.C. Fang, Passively Q-switched erbium all-fiber lasers by use of thulium-doped saturable-absorber fibers, Opt. Express 18 (2010) 10049, http://dx.doi.org/10.1364/OE.18.010049.

[21] M. Tao, J. Wu, J. Peng, Y. Wu, P. Yang, X. Ye, Experimental demonstration of an er-doped fiber ring laser mode-locked with a Tm-Ho co-doped fiber saturable absorber, Laser Phys. 23 (2013) 3136-3141, http://dx.doi.org/10.1088/1054$660 x / 23 / 8 / 085102$
[22] A. Latiff, N. Kadir, E. Ismail, H. Shamsuddin, H. Ahmad, S. Harun, All-fiber dualwavelength Q-switched and mode-locked EDFL by SMF-THDF-SMF structure as a saturable absorber, Opt. Commun. 389 (2017) 29-34, http://dx.doi.org/10. 1016/j.optcom.2016.12.011.

[23] S.D. Jackson, The spectroscopic and energy transfer characteristics of the rare earth ions used for silicate glass fibre lasers operating in the shortwave infrared, Laser Photon. Rev. 3 (2009) 466-482, http://dx.doi.org/10.1002/lpor. 200810058.

[24] P. Zhang, T. Wang, W. Ma, K. Dong, H. Jiang, Tunable multiwavelength Tmdoped fiber laser based on the multimode interference effect, Appl. Opt. 54 (2015) 4667-4671, http://dx.doi.org/10.1364/AO.54.004667.

[25] H. Yu, X. Zheng, K. Yin, T. Jiang, Thulium/holmium-doped fiber laser passively mode locked by black phosphorus nanoplatelets-based saturable absorber, Appl. Opt. 54 (2015) 10290-10294, http://dx.doi.org/10.1364/ao.54.010290.

[26] M. Tao, G. Feng, A. Andrianov, A. Kim, T. Yu, H. Zhao, X. Ye, Tm-ho codoped fiber based multi-wavelength Q-switching of an Er-doped fiber laser, Opt. Commun. 354 (2015) 209-212, http://dx.doi.org/10.1016/j.optcom.2015. 05.060 .

[27] M. Tao, X. Ye, Z. Wang, Y. Yan, G. Feng, P. Yang, G. Feng, Modeling of TmHo codoped fiber saturable absorber based passive Q-switching of an Er-doped fiber laser, Laser Phys. 24 (2014) 085110, http://dx.doi.org/10.1088/1054$660 X / 24 / 8 / 085110$.

[28] T. Jiang, Y. Xu, Q. Tian, L. Liu, Passively Q-switching induced by gold nanocrystals, Appl. Phys. Lett. 101 (2012) 151110, http://dx.doi.org/10.1063/ 1.4759120.

[29] D. Zhao, G. Qin, L. Liu, W. Qin, X. Guo, Y. Xu, Z. Kang, Z. Jia, Gold nanorods as saturable absorbers for all-fiber passively Q-switched erbium-doped fiber laser, Opt. Mater. Express 3 (2013) 1986-1991, http://dx.doi.org/10.1364/OME.3. 001986. 